\title{
Contract Research in Design
}

\author{
REVISED DRAFT 28.05.03 \\ Published in Design Issues, 20(4): 34-43
}

(Originally submitted as a conference paper and published in the proceedings of "Common Ground”, Design Research Society Conference, Brunel University, September 2002)

Anthony Crabbe, Leader, Design Contract Research Unit, Nottingham Trent University, Burton Street, Nottingham

NG1 4BU

Tel direct: 01158482087

anthony.crabbe@ntu.ac.uk

\begin{abstract}
Contract research, which is a commercial research service undertaken for commissioning clients, may be seen to amount to little more than jobbing work. This paper considers case studies undertaken by my own university unit in light of various theoretical positions about research as a knowledge building activity, paying particular attention to the notions of innovation characterised in patent regulations. The review suggests that design research into a single area, such as our case study of rotationally moulded products, typically involves the skills of project management, with a greater requirement for broad knowledge of a number of research methodologies, rather than just one that is especial to design research. These reflections may suggest that a shared understanding of design research is as well assisted by retrospective examination of practice, as by prospective theoretical debate.
\end{abstract}

Keywords: Research, Knowledge, Design Practice, Rotational Moulding

Contract research is a commercial research service undertaken for commissioning clients. This activity may pose difficult questions for researchers in the design area, since a commercial service may appear capable of producing little more than jobbing practice. The present paper considers case studies of work carried out by the Design Contract Research Unit at Nottingham Trent University in light of various theoretical positions on research. The aim of the discussion is to better clarify the controversial relationship of commercial design practice to what is now coming to be recognised as design research.

$\underline{\text { Research and Practice }}$

In the UK, there is political pressure on academic communities to reach consensus about the nature and value of research in their chosen disciplines, most obviously 
evidenced in the introduction of national Research Assessment Exercises. Politicians and civil servants seem increasingly drawn to the idea of fixing an apparently tangible value on the quality of public activity by creating new funding equations. An audit like the RAE is a useful means of demonstrating their diligence and the accountability of their fund management. However, success in such an exercise is not the beginning or end of funding support for design research. Design is an element of industrial culture and some of the most impressive research campuses to be found are those belonging to giant industrial corporations, such as Microsoft and Nestlé. Armies of researchers also inhabit those campuses and it would be a serious misunderstanding in those of us less well accommodated in universities to believe that somehow, our industrial colleagues are working one level below us, tied as they are to the directions of greedy masters. Consider that such masters may provide academics with patronage additional to that given by politicians and bureaucrats, whose motives (such as maintenance of personal office) are not obviously purer.

Some may argue that the outcomes of commercial research and development evidence 'applied' research, which seems by implication to be a rung down the ladder from 'pure' research. A less specious distinction to make is that between research with a pre-determined goal, and research without the same (which is often called 'fundamental' research in the sciences). An example of the former would be to find a way of preventing a carbon filament that becomes incandescent when an electrical current passes through it, from burning up after a few seconds. This was a major research project that led to the invention of the first durable electric light bulb by Edison, using a largely empirical trial and error method.

An example of fundamental research would be to investigate what happens when electrical currents are passed through strands of different materials. In hindsight, this may seem a necessary precursor for inventing a light bulb, but in foresight, it does not appear to be a research programme guaranteed to add even to theoretical understanding of electromagnetic behaviour. It is invidious to value one approach more highly than another. Both exist in design research, yet goal-led research is evidently the more dominant form because research programmes can be very expensive and so market forces in both public and private sectors favour the goal-led form in design. Indeed, it is hard to imagine that design researchers could learn much of value from practice-based activity unless there were commercial manufacturers and developers available to collaborate in essential realisation processes, such as tooling, fabrication and distribution. It is largely due to this consideration that my own unit has been led into accepting goals set by clients, rather than ourselves, and why the term 'contract' prefixes our research activity.

Concerning the notion of practice in relation to research, Nigel Cross is persuasive in insisting that practice itself does not constitute a significant research activity, because in a community, others may feel that if they cannot gain access through public reports to the methods behind the outcome, they cannot easily assess their value or further applicability ${ }^{1}$. In the case of craft production, many craftspeople would probably go to considerable lengths not to disclose their methods to others. The success of such an approach both in defending innovation as well as adding value or mystique to the products is well evidenced by the successful transition of famous Renaissance figures, such as Leonardo, from the status of craftsman to artist. Parallels are still to be found in contemporary design, where the status of designers such as Armani and Starck, 
indicates that even in an industrial culture, mystique still plays an important role in the value systems of consumers, and profit margins of marketers.

More commonly in industrial cultures, we have mechanisms for protecting personal innovation by actually disclosing outcomes in formal public ways. Patent and copyright are the most obvious examples and both are recognised as satisfactory research outcomes by UK research assessment exercises. Patents must by definition be: 1) new ideas, not previously disclosed in public, 2) involve an inventive step, such that 'when compared with what is already known, it would not be obvious to someone with a good knowledge and experience of the subject', 3) 'be capable of industrial application'. ${ }^{2}$ In this respect, 'industry is meant in its broadest sense as anything distinct from purely intellectual or aesthetic activity'. Under such definition, natural discoveries, scientific theories, mathematical methods and aesthetic creations are excluded from patent protection. On the other hand, the specific form of an aesthetic creation, such as the exact words of a text, or the patterns and shapes of a designed object can be protected under copyright or design patent.

Patent definitions are then most instructive in telling us about the forms of knowledge which are pertinent to the definition of design research. Design practice primarily concerns the creation of apparatus, devices, processes or methods of operation that are capable of industrial application. Whilst it is by no means necessary that the outcomes of design practice are in any way inventive, many of them may be claimed to take a specific form that is novel and can be disclosed and protected. The ordinary patent involves creating products, methods or processes, which can be described in such a way as to enable others to reproduce and apply the inventive steps. The design patent involves creating a specific arrangement of symbols, shapes, lines or patterns, which so differs to precursors, that just describing it in patent form prevents others from trying to reproduce the arrangement without permission. Of the two kinds of disclosure, the ordinary patent makes it far easier for others to gain insight into the particular research and creative processes giving rise to the outcome. Designers, like other professionals, may then wish to comment publicly through means such as conference or publication, on the kinds of approaches and insights underlying particular design outcomes. This constitutes a third form of contribution to public knowledge, which is not patentable, but is recognised as a vital part of the research culture of any discipline.

\section{Research and Knowledge}

As to the relationship between research and knowledge, the dictionary definitions of research include 'collecting information about a subject', in a way that is 'careful or diligent'. This diligent way may also involve a more complex 'investigation and experimentation aimed at the discovery and interpretation of new facts, revision of accepted theories or laws in the light of new facts, or practical application of such new or revised theories or laws'. ${ }^{3}$

An attribute of research in general that is embedded in the official guidelines of organisations like the UK RAE is that it 'contributes to knowledge'. In this sort of description, knowledge seems to be principally the public kind, and accordingly, a contribution may be seen as something that is new, or different enough, to add to a public 'bank' of knowledge. For patents there is a highly developed and complex method that allows professional examiners to determine the extent to which knowledge claims may be deemed new additions. Unfortunately, for forms of 
knowledge 'excluded' from patenting, such as intellectual discoveries and theories, it is far less clear cut how they come to be accepted as additions. The primary mechanism is that of peer review by academics, publishers and media editors.

As a relatively young and emergent discipline, design introduces problematic issues of its own. There seems to be consensus that design is very much an interdisciplinary activity, attracting inward a variety of research paradigms from longer established academic disciplines. ${ }^{4}$ There also seems to be some agreement even between those with differing views of design research, such as Charles Owen ${ }^{5}$ and Ezio Manzini ${ }^{6}$ that it is right and proper for all those different specialists gathered under the design umbrella to develop new research paradigms.

Among the new paradigms entering design, is post-structuralism, or 'the new criticism ${ }^{7}$ which challenges traditional knowledge hierarchies. Although most evidenced in what used to be called literary criticism, the new approach is derived from the work of cross disciplinary mentors like the psychoanalyst Jacques Lacan and the philosopher Jacques Derrida. Derrida argues that no form of knowledge is 'centred', there is no unique 'logos' or knowledge structure that is truer than any other. ${ }^{8}$ In fact, Derrida's main point here has already been expressed by other philosophers, as different as Karl Popper and Richard Rorty. Popper has argued that knowledge comprises a network of theories, in which even the firmest beliefs appear to be provisional, subject to the discovery of a better theory. ${ }^{9}$

Rorty attacks the 'foundational' view of knowledge, where philosophers have traditionally assumed a privileged view of knowledge in general, which portrays different forms of knowledge building up from a hard base layer of the cognitive kind to progressively softer layers of the hermeneutic kind. ${ }^{10}$ Popper seems to be one of these traditional philosophers, arguing that objective knowledge, such as "The Earth orbits the Sun”, holds a special place, because the veracity of such propositions does not appear to depend on subjective choice. Objectivity is clearly an important feature of the way knowledge is viewed in the hard sciences and may help to explain why even great creations such as relativity theory are more usually described as "discoveries". As recognised in the earlier discussion of patenting, design activity may involve some form of new discovery that can be tested in a way that provides reproducible results. However, design also encompasses forms of creative output which can be recognised, described and evaluated, but only in the form of a critical activity that appeals to a sharing of personal experiences and aesthetic codes.

It is unlikely that many in design would want to claim that critical arguments impose the same sense of necessity on the understanding as do objective findings about, say, the physical performance of designed objects. Accordingly, design by its very nature seems divided between views of knowledge that differ according to the kind of activity undertaken and questions posed. Designers are frequently called upon to tackle different problems, which involve different forms of knowledge and thus, methodology. For instance, the writing of this paper involves critical discourse, which appeals to subjective experience, leaving the arguments open to a spectrum of personal interpretations. Whereas, some of the product design work I am about to discuss is not open to the same level of subjective interpretation, it either performs to an International Standard, makes valid patent claims, or it does not - and these issues can be resolved by reproducible testing and examination. Such work is not even typical of much product design, which concerns re-styling familiar objects, an activity 
that could be the subject of a design patent, but not an ordinary one. Differing research methodologies are bound to underlie such different tasks, and anyone working on three such projects is bound to adjust their goals, knowledge claims and research methods, without ceasing to be engaged in some form of worthwhile design research.

However, caution needs to be exercised in the selection of research methodologies and paradigms, as evidenced by the example of some of the new criticism. Consider the "Theory of the Gaze”, originated by Laura Mulvey’s 1975 article "Visual Pleasure and Narrative Cinema". ${ }^{11}$ This has been a very influential critical stance on film narrative, based on an entirely uncritical acceptance of Freud's theory of scopophilia, which although probably new to most in visual studies at the time, was already regarded as outmoded and unreliable by many in psychology. ${ }^{12}$ When introducing ideas and methods from contingent disciplines. It is better advised to select from them the methodologies that seem most appropriate to particular tasks. Through informed adaptation to specific requirements in design, existing methodologies may even be revised or expanded to become generally useful in design research. For instance, asking if a design is patentable is a useful way of assessing the sense in which workers from all disciplines may see it to be innovative, but not of regarding it critically.

Returning to the value of patents as indicators of worthwhile research activity, if numbers demonstrate anything, our colleagues in the corporate sector are making a far more prolific contribution in the field of product innovation than ourselves. However, to recognise this is not to exclude academic researchers from the field. There are many small to medium enterprises (SMEs) who cannot afford to maintain their own research and development units. To such organisations, universities can offer what in today's parlance is called 'knowledge transfer'.

In many countries this transfer can be supported by state funding initiatives. We have been supported by European Regional Development Funding, with a remit to provide a subsidised knowledge transfer service to SMEs. This imposes the condition that we should not therefore be competing for work with local design agencies. Academics have the benefit of being part of a much larger expert community whose presence greatly increases the range of methods, techniques and resources we can bring to bear in planning a goal-led research programme. Few agencies either could or would want to compete with these resources and so that makes it easier for us to identify the kind of projects in which we want to get involved. Our rule of thumb is that we say "sorry" to any company asking us to "Design one of those", but welcome collaboration with anyone asking us "Do you think it would be possible to design something that...?", or “Are we going the right way about designing this?” A good demonstration of this principle is provided by our case study, a collaboration with a small, but successful plastics company in our catchment area.

\section{Case Study: Design for Rotational Moulding}

Europalite Ltd. mould plastic products like road cones and grit bins by rotational methods. Essentially a rotationally moulded form is a single plastic surface bounding a closed volume - a hollow sphere is a typical primitive. On the other hand, an open form like a bowl is not typical, but could be made by cutting a rotationally moulded sphere in half. The process also allows more complex primitives, such as a form pierced through by a hole - "genus 1" in mathematical language - as well as genus 2, 
3 , and so on, provided the walls of the holes are all orientated on the same axis and do not "return" into the body of the basic form. Whilst the method is less flexible than other moulding processes in allowing a variety of geometries, plastic affords more opportunities for constructing complex forms than kindred processes such as clay slip casting. The vast majority of moulds are split into two parts, which are filled with finely ground plastic, sealed and then rotated bi-axially in a large oven that causes the polymer to melt and attach to the wall surfaces inside the mould, which may later be split open to release the finished product. The two great advantages of rotational moulding are that it can produce large products, and the mould tools are cheap to fabricate or cast, typically costing between $10-25 \%$ the price of much smaller injection tools. It is then, a relatively simple process, often associated with large utilitarian products of relatively low production quality, and large tolerances of accuracy.

The managing director contacted us because he thought the process was capable of far more than his industry has demonstrated thus far. Early in our association, he suggested to that we investigate the possibility of designing an adjustable builder's trestle to compete with the tubular steel variety that are fabricated to meet stringent British Standards in safely supporting a working load of 650kg (BS 1139:4:1982). His cheerful justification of why he should want to attempt such a project was, "Because I make things in plastic". Whilst this had scared away design agencies, it proved irresistible bait to people who enjoy getting their students to build improbably strong bridges out of drinking straws. It was a project through which we felt we could learn, and this made it seem an ideal form of knowledge transfer.

For this project we purchased a basic Finite Element Analysis (FEA) package, Design Space, not only to assist the design process, but to evaluate an application, which in principle should be usable by product designers, not just trained engineers. Having generated a number of concept designs (Figure 1), some were input into Design Space, which grudgingly started to give answers to the engineering questions (Figure 2). It was not until the project was almost completed that we discovered we had been asking the program to do more than it was designed to, in analysing hollow forms, rather than solids. We cross-checked the FEA solutions by taking small segments of a given part and calculating the answers manually. Then a prototype fabricated steel tool was made of the selected final design.

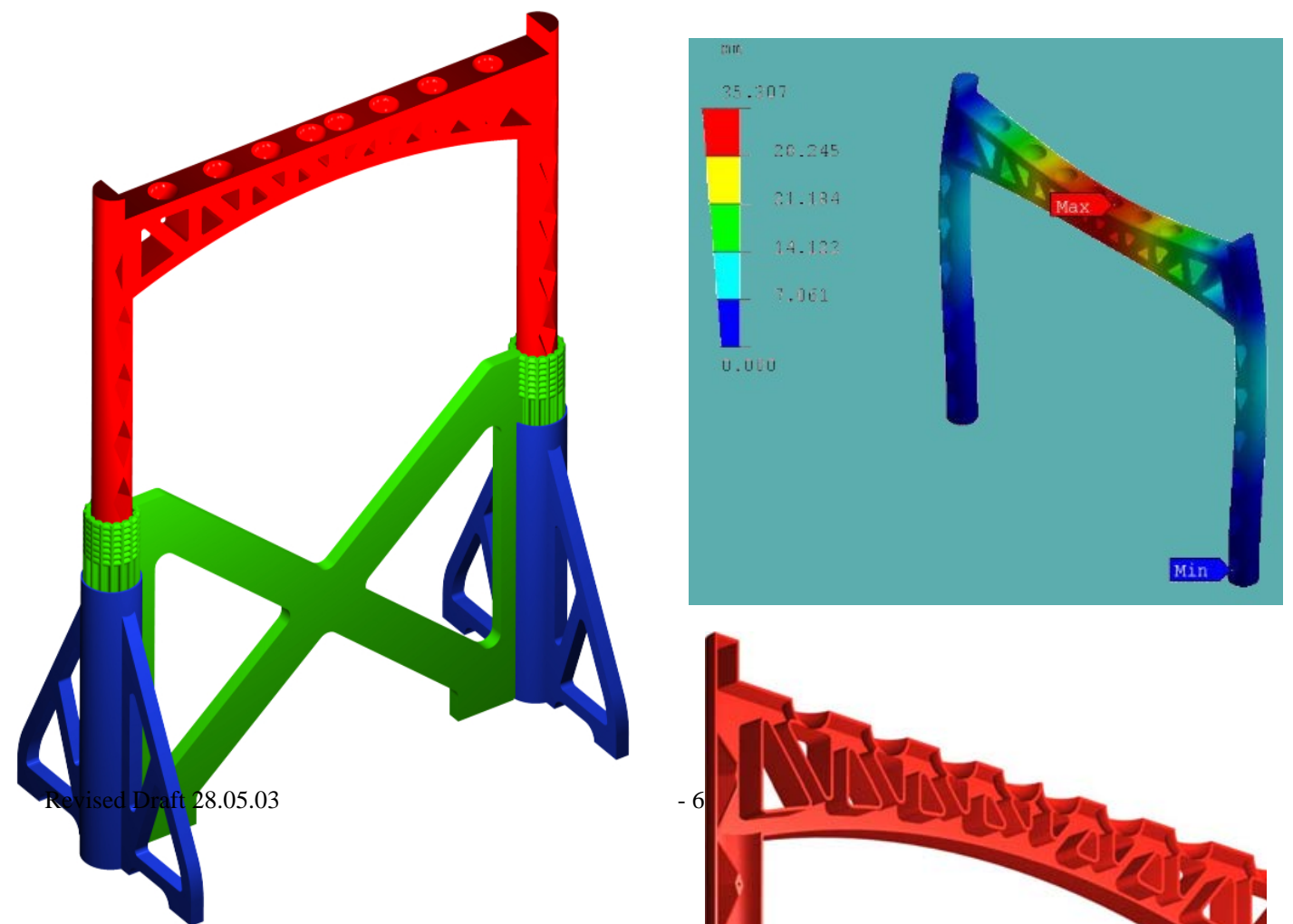


Figure 1 Builders' trestle

Figure 2 Finite element analysis of trestle

Figure 3 Section through trestle beam

In the event, the loading simulations were within $12 \%$ of the real values found in the final design. From this we concluded that the latest FEA applications should be viable tools for product designers without a formal engineering training. Despite our reservations about the eventual commercial viability of the trestle, we sought throughout the project to play to the strengths of a moulded trestle, by limiting the components to four forms that could be inexpensively moulded with few fabrication steps thereafter, and assembled from a flat pack by the user. The standards testing we were able to contract in house from our engineering laboratories and the dissemination of what we had learnt was in part publicised through the filing of a patent.

Another vital part of our mutual learning was an investigation into whether it was possible to increase the strength of the polyethylene polymer we were using, perhaps by glass fibre reinforcement. This investigation demonstrates the value of patent literature to design researchers, since we found two patents from the 1980s, which showed the polymer suppliers to be wrong in their assertion that rotationally moulded plastics could not be successfully glass reinforced. When we tried to replicate the methods disclosed in the patents, the results showed the fibre tended to migrate into the inside of the product walls and was poorly packed, which made the strength of the compound less than expected in comparison to other moulding methods. Proceeding in a way more reminiscent of Edison's empirical approach to the light bulb than of contemporary polymer engineers, we guessed that the problem lay in the glass strand dimensions. So we got samples of several non standard strand types, to compound in a variety of different test batches. We were fortunate in achieving the desired result of a greatly strengthened product within a few hours of moulding.

The next task was to further improve the strength of the glass to polymer bond by finding a more appropriate chemical coupling agent than those described in the patents. Despite superb support and advice from Akzo Nobel and Hoechst, we had far greater difficulty in these tests. The eventual solution was again derived from a leap of designer's intuition, rather than formal analysis. We felt an instinctive discomfort in suggesting that the workforce made up the moulding compound with a rather hazardous liquid chemical. This led to a search for a powder based form, which we could not find, but we did come across an analogue product used in rheology, rather than coupling, that was based in a fine chalk powder of similar grain size to the polymer. Again, we used empirical methods to find the correct concentration, and the strengthening effect was so tangible we scarcely needed laboratory testing to tell us which measure and mixing method gave us the best coupling. The results of this work are to be disclosed in another patent file.

The final example concerns a project more within our range of expertise, which arose from the company's success in persuading us that there was untapped potential in rotational moulding. The problems to be overcome had more to do with the standards of toolmaking, than of product design. The tolerances of steel fabricated mould tools are at least $2 \mathrm{~mm}$ over $1 \mathrm{~m}$ and wall thickness can vary up to $20 \%$. In theory, an aluminium tool cast from a wooden model, or pattern, can be made accurate to 
fractions of a millimetre, but then the patterns are hand built from the design drawings and therefore prone to larger error. In the trestle, we had to connect opposing walls in the hollow form to create a true structure, rather than a void enclosed by unconnected walls. This we did by dimpling key areas of the walls to create "kiss points" inside the form as the product moulded (Figure 3). The unconventional dimple forms we created did not endear us to the toolmakers, whose notions of engineering tolerance did not endear them to us. If we could find a more accurate way of generating the patterns, we felt we could overcome the limitations of the process to liberate its potentials.

These are that the ovens can be as large as 4 metres in diameter, which means smaller products can be tooled as "parasites" that are just fixed into any space not filled by a larger product being moulded. Given tooling costs of as little as £2-3000 for a product the size of a torch, the parasites can act as prototype generators, which if successful, can be duplicated and arrays of these small products can be moulded 10 or 20 at a time, for a fraction of the cost of an injection moulding tool manufacturing them at a comparable rate. So here, rather than trying to apply rotational moulding to products never made before in plastic, we were seeking to advance rotational moulding into a more competitive form of making plastic products. A good vehicle for this idea turned out to be a hard hat, a product always injection moulded, with a typical tooling cost of $£ 80-100,000$. Since a hard hat is essentially a shell supported by an adjustable webbing cradle, we set out to see if it were possible to turn the underside of a rotationally moulded hat into a webbing and find an alternative method of adjusting the headband to fit all sizes of head (Figure 4).

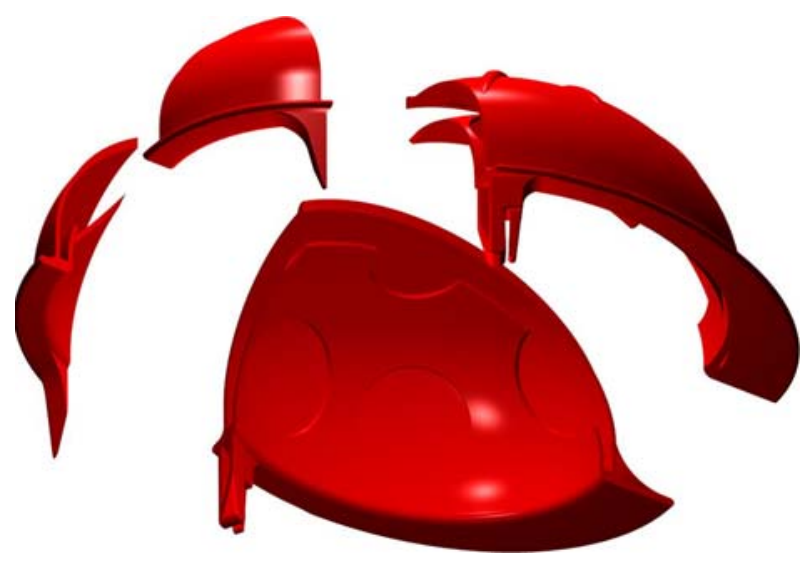

Figure 4 Exploded view of hard hat

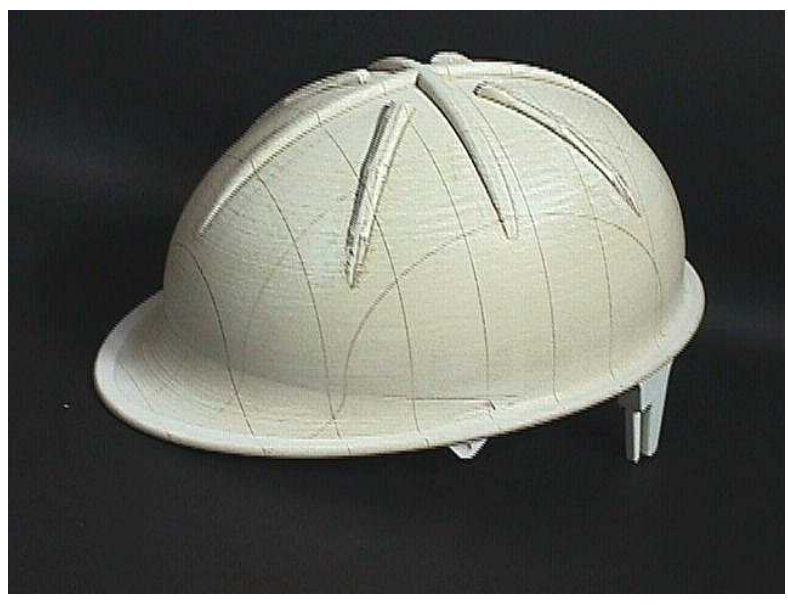

Figure 5 Rapid prototype of hard hat

The design solutions seemed relatively simple. The adjustable back of a baseball cap shows how a broad range of hat size adjustment could be made by attachment of a belt-type strap to two small lugs on the rim. Whilst the webbing could be created by cutting the shapes of the holes in the webbing from a low conducting material, and then fixing these cut-outs on the relevant surfaces of the mould tool, so that no plastic would form on them. The real problem was how to ensure the accuracy of tooling that was essential from a structural point of view and indeed an aesthetic one, because this was an apparel item. Construction workers seem to have a greater consciousness of their appearance than may be imagined. Evidence comes in the form of the novelty Stetson hard hat, which is apparently a major seller in the US heartlands. The fact that our hat has ribs which form a Union Jack is completely fortuitous, a result of our mainly structural approach to the task. Nevertheless, we were perfectly happy to 
exploit this accident and have only half-jokingly suggested that the client might consider marketing it in the UK as the "Jack Hat".

As to making an acceptably accurate model, we turned to colleagues in Nottingham University to help us rapid prototype an extremely accurate solid model direct from our original CAD files. For this relatively small product it was economically acceptable to use LOM, laminated object manufacture, which produces the "wood" model by scanning the CAD model in paper thin horizontal slices and then laser cutting the slice from a sheet of paper, running a glue impregnated roller over the slice and then repeating to generate the complete model (Figure 5). Plaster patterns were taken from the model, from which the aluminium mould tool parts could be sand cast. The tool casting is taking place at the time of writing, so the results are not yet fully known. However, we are confident that our approach is the way forward to realising the larger objective of introducing rapid, accurate tool-making straight from the designer's CAD files in order to facilitate a new generation of products that conform both to consumer expectation and to necessary regulatory standards for public health and safety.

\section{Conclusion}

As to the lessons that can be learnt from the practice of contract research in design, the following seem instructive: Unlike art or craft activity, professional design generates plans that are seldom realisable by the designer and require the application of technologies and resources largely controlled by third party commercial enterprises. Those seeking to develop a consensual view of design research should not then overlook the real relationship of design with commerce. Commercial imperatives clearly impact on designers' research approaches as well as their practice. It may appear from the case studies that goal-led research for commercial clients encourages less inhibited methodology, because the primary goal is research that produces a tangible commercial effect. Yet whilst ends very much justify means in contract research, they do not necessarily handicap good design research. The design researcher need not have all the expertise required by the project, good project management skills are more appropriate, and key among these is the ability to recognise what expertise and methodologies need to be introduced from outside the discipline. This suggests that breadth of knowledge is an important characteristic of both design researcher and practitioner, and further implies that depth of specialist knowledge may not count as much as in other disciplines.

We have found that the effect on clients of working with academic researchers is to liberate their risk-taking and playfulness, which are vital ingredients both for creativity and formulating interesting research questions. Play involves a considerable element of trial and error, an approach that may have become rather unrespectable in formal scientific research, but is very much part of the designer's working method especially since product design involves speculative activities such as criticism, which appears to play little or no role in the practice of 'hard' science. Some of the research methods described in our case study might appear too informal to purely academic researchers. We defend the methods on the basis that the contracted goals were achieved, allowing new products to be realised and their designs appropriately reported and disseminated, for instance, through patent files. These reflections may suggest that a shared understanding of design research is as well assisted by retrospective examination of practice, as by prospective theoretical debate. 


\section{REFERENCES}

${ }^{1}$ N. Cross, 1999, “Design Research: A Disciplined Conversation”, Design Issues 15:2, (1999) 5-10

2 UK Patent Office, www.patent.gov.uk (2002)

3 Britannica Webster's, Encyclopaedia Britannica Online (www.britannica.com, 2002)

${ }^{4}$ V. Margolin, “Design Research and its Challenges” Design Journal 15:2 (1999) 1419

${ }^{5}$ C. Owen, "Design Research: Building the Knowledge Base” Design/Research Conference Paper, (London: Royal College of Art, 1994)

${ }^{6}$ E. Manzini, "Design Research for a Sustainable Environment” Design/Research Conference Paper, (London: Royal College of Art, 1994)

${ }^{7}$ A. Seago \& A. Dunne, "New Methodologies in Art and Design Research: The Object as Discourse”, Design Issues 15:2 (1999) 11-17

${ }^{8}$ J. Derrida, Margins of Philosophy (Brighton: Harvester 1982)

${ }^{9}$ K. Popper, 1972, Objective knowledge : an evolutionary approach (Oxford: Oxford University Press 1972) 71-81

${ }^{10}$ R. Rorty , Philosophy and the mirror of nature (Oxford: Blackwell, 1980) 313-22

${ }^{11}$ L. Mulvey, "Visual pleasure and narrative Cinema" Screen, XV1 no 3. (1975)

${ }^{12}$ J. Eysenck \& G.D. Wilson, The experimental study of Freudian theories (London: Methuen, 1973) 1-13 